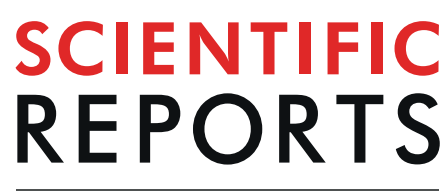

natureresearch

Check for updates

\title{
Pivotal role of CD103 in the development of psoriasiform dermatitis
}

\author{
Takehito Fukui ${ }^{1,2}$, Tomohiro Fukaya ${ }^{1,7}$, Tomofumi Uto ${ }^{1,7}$, Hideaki Takagi ${ }^{1,7}$, Junta Nasu ${ }^{1,2}$, \\ Noriaki Miyanaga ${ }^{1,3}$, Yotaro Nishikawa ${ }^{1,4}$, Haruhiko Koseki ${ }^{5}$, Narantsog Choijookhuu ${ }^{6}$, \\ Yoshitaka Hishikawa ${ }^{6}$, Yoshihiro Yamashita ${ }^{2} \&$ Katsuaki Sato ${ }^{1,7 凶}$
}

The integrin $\alpha E$ known as CD103 binds integrin $\beta 7$ to form the complete heterodimeric integrin molecule $\alpha E \beta 7$. CD103 is mainly expressed by lymphocytes within epithelial tissues of intestine, lung, and skin as well as subsets of mucosal and dermal conventional dendritic cells (CDCs). CD103 has been originally implicated in the attachment of lymphocytes to epithelium in the gut and skin through the interaction with E-cadherin expressed on intestinal epithelial cells, keratinocytes, and Langerhans cells (LCs). However, an impact of CD103 on the cutaneous immune responses and the development of inflammatory skin diseases remains elusive. Here, we report that CD103 regulates the development of psoriasiform dermatitis through the control of the function of CDCs. Deficiency in CD103 exacerbates psoriasiform dermatitis, accompanied by excessive epidermal hyperplasia and infiltration of inflammatory leukocytes. Furthermore, deficiency in CD103 not only accelerates the production of proinflammatory cytokines in psoriatic lesions but also promotes the generation of lymphocytes producing interleukin (IL)-17 in the skin-draining peripheral lymph nodes (PLNs). Under the deficiency in CD103, cDCs localized in PLNs enhance cytokine production following activation. Thus, our findings reveal a pivotal role for $\mathrm{CD} 103$ in the control of the function of CDCs to regulate cutaneous inflammation in psoriasiform dermatitis.

Dendritic cells (DCs) are essential antigen (Ag)-presenting cells (APCs) to produce multiple cytokines and activate naïve $\mathrm{T}$ cells during primary responses upon recognition of the pathogens through pattern recognition receptors (PRRs) ${ }^{1-3}$. Therefore, DCs play critical roles in orchestrating the immune system, linking innate and adaptive immunity ${ }^{4-6}$. DCs are classified into two major subsets, classical or conventional DCs (cDCs) and plasmacytoid DCs (pDCs) ${ }^{1-3}$. cDCs display an unique ability to prime naïve T cells to induce various types of effector $\mathrm{T}\left(\mathrm{T}_{\mathrm{eff}}\right)$ cells due to the remarkable expressions of major histocompatibility complex (MHC) molecules and costimulatory molecules ${ }^{1-3}$. Thus, $\mathrm{cDCs}$ has been recognized as central to regulate the direction of adaptive immune responses for the protection against microbial infections. On the other hand, pDCs are characterized to secrete the massive amounts of type I IFN (IFN-I) following recognition of viral nucleic acids (NAs) through endosomal TLR7/94. Therefore, pDCs have been considered as critical immune cells of antiviral responses.

Psoriasis, one of the most common chronic inflammatory cutaneous disease affecting around $2 \%$ of the global population, is characterized by several pathological features including red, scaly, raised plaques at different body sites, finally causing the impairment of the skin barrier function ${ }^{5-8}$. Histological manifestations of psoriasis include diffuse epidermal hyperplasia (acanthosis) owing to hyperproliferation of keratinocytes and parakeratosis that is caused by an aberrant differentiation of keratinocytes ${ }^{8}$, as well as prominent inflammatory

${ }^{1}$ Division of Immunology, Department of Infectious Diseases, Faculty of Medicine, University of Miyazaki, 5200 Kihara, Kiyotake, Miyazaki, 889-1692, Japan. ${ }^{2}$ Department of Oral and Maxillofacial Surgery, Faculty of Medicine, University of Miyazaki, 5200 Kihara, Kiyotake, Miyazaki, 889-1692, Japan. ${ }^{3}$ Department of Otolaryngology, Head and Neck Surgery, Faculty of Medicine, University of Miyazaki, 5200 Kihara, Kiyotake, Miyazaki, 889-1692, Japan. ${ }^{4}$ Department of Dermatology, Faculty of Medicine, University of Miyazaki, 5200 Kihara, Kiyotake, Miyazaki, 8891692, Japan. ${ }^{5}$ Laboratory for Developmental Genetics, RIKEN Center for Integrative Medical Sciences, 1-7-22 Suehiro-cho, Tsurumi-ku, Yokohama, Kanagawa, 230-0045, Japan. ${ }^{6}$ Division of Histochemistry and Cell Biology, Department of Anatomy, Faculty of Medicine, University of Miyazaki, Miyazaki, 889-1692, Japan. ${ }^{7}$ Japan Agency for Medical Research and Development (AMED), 1-7-1 Otemachi, Chiyoda-ku, Tokyo, 100-0004, Japan. ${ }^{凶}$ e-mail: katsuaki_sato@med.miyazaki-u.ac.jp 


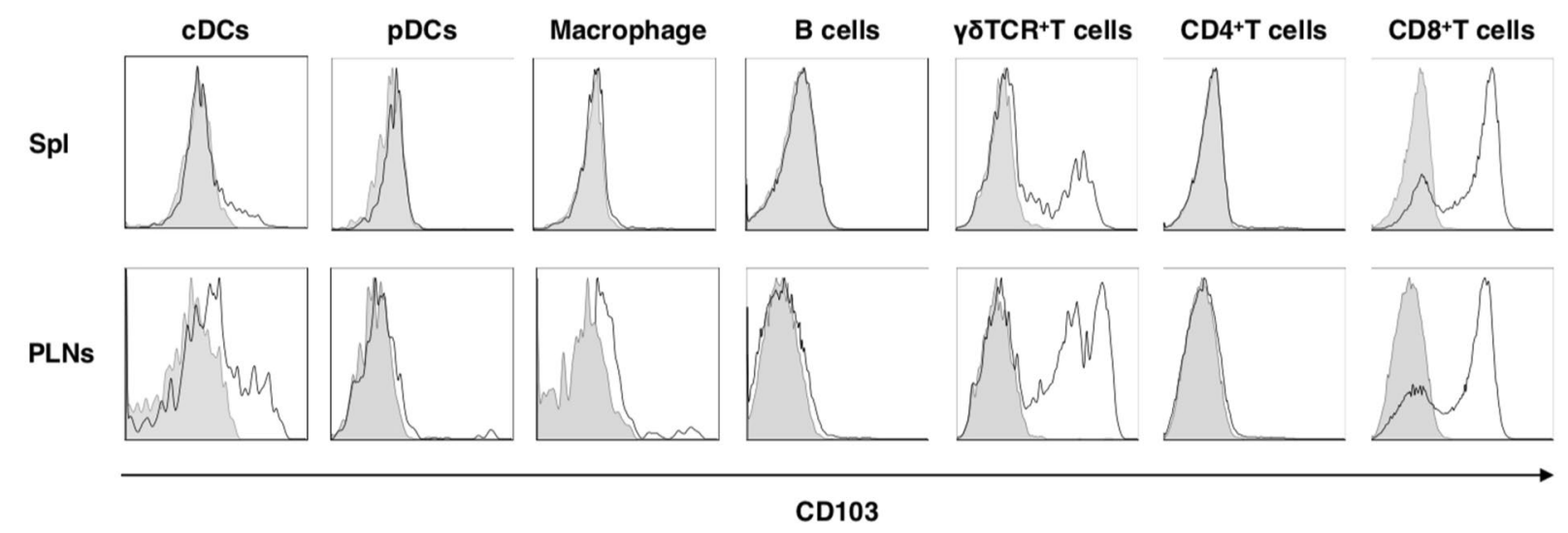

Figure 1. The expression of CD103 on leukocytes in lymphoid tissues. The expression of CD103 on leukocytes derived from WT mice and $C d 103^{-1-}$ mice. Data are presented by a histogram, in which open or gray indicate WT mice or $C d 103^{-1-}$ mice, respectively. The results are representative of at least three independent experiments.

infiltrating leukocytes consisting of T cells, macrophages, and DCs in the dermis and neutrophils in the epider$\mathrm{mis}^{9-11}$. Whereas several inflammatory cytokines such as interleukin (IL)-1 $\beta$, IL-6, and tumor necrosis factor (TNF)- $\alpha$, and type I interferon (IFN-I) have been associated with the priming and skewing of psoriatic inflammation $^{11,12}$, recent studies have shown that the axis of IL-22-IL-23-IL-17 cytokine is critical for the development of skin immunopathology ${ }^{13-16}$. While the exact initiation process for psoriasis is not fully understood, IL-23 secreted by macrophages and cDCs induces the expansion and activation of T helper $17\left(\mathrm{~T}_{\mathrm{H}} 17\right)$ cells, $\mathrm{T}$ cytotoxic $17\left(\mathrm{~T}_{\mathrm{C}} 17\right)$ cells, and $\gamma \delta \mathrm{T}$ cells, as well as type 3 innate lymphoid cells (ILC3) to produce IL-17 and their related cytokines in the process of psoriatic skin inflammation ${ }^{9,13-16}$.

The integrin $\alpha E$ (ITGAE) known as CD103 binds integrin $\beta 7$ (ITGB7) to form the complete heterodimeric integrin molecule $\alpha \mathrm{E} \beta 37^{17-19}$. CD103 is primarily expressed by epithelial T cells, including CD $4^{+}$and $\mathrm{CD} 8^{+} \mathrm{T}$ cells and $\gamma \delta \mathrm{TCR}{ }^{+}$ $\mathrm{T}$ cells ${ }^{17-20}$, as well as other leukocytes such as a population of $\mathrm{CDCs}^{1-3}$ in the intestine, lung, and skin. CD103 has been originally implicated in the attachment of lymphocytes to epithelial tissues in the gut and skin through the interaction with E-cadherin expressed on intestinal epithelial cells, keratinocytes, and Langerhans cells (LCs) ${ }^{17-20}$. In several experimental models with CD103-deficient mice, CD103 has been required for the localization of CD8 $\alpha^{+}$intraepithelial lymphocytes (IELs) bearing $\alpha \beta T$-cell receptor (TCR) in the gut $^{21}$ and epidermal $\gamma \delta \mathrm{TCR}^{+} \mathrm{T}$ cells in the skin ${ }^{22}$. CD103 has been involved in the destruction of pancreatic islet allografts ${ }^{23}$ and intestines in graft-versus-host diseases (GVHD) ${ }^{24}$ through the promotion of the migration of the pathogenic $\mathrm{CD}^{+} \mathrm{T}$ cells into epithelial compartments. Furthermore, it has been shown that CD103 contributes to the development of airway inflammation in asthma ${ }^{25}$. On the other hand, CD103 is dispensable for the control of a chronic antiviral immune response ${ }^{26}$ and intestinal immunity to helminth infection $^{27}$. In the skin pathogenesis, CD103 has reportedly participates in the development of allergic contact hypersensitivity through the regulation of the retention of $\mathrm{CD}^{+}{ }^{+}$Foxp $3^{+}$regulatory $\mathrm{T}\left(\mathrm{T}_{\text {reg }}\right)$ cells to the inflamed $\operatorname{skin}^{28}$ as well as the induction of cutaneous inflammatory disorder, that was affected by some environmental conditions and/ or in the context of other genetic factors ${ }^{20}$. While CD103 has reportedly no obvious pathogenic role in psoriasiform skin lesions of transgenic (Tg) mice overexpressing human transforming growth factor (TGF)- $\beta 1$ under the regulation of the keratin 5 promoter within the epidermis (K5.hTGF $31 \mathrm{Tg}$ mice), which displayed a spontaneous development of psoriatic dermatitis ${ }^{29}$, the potential role of CD103 in the control of the pathogenesis of psoriasiform dermatitis and other cutaneous inflammatory disorders remains elusive.

In this study, we show that impact of CD103 on the development of psoriasiform dermatitis mediated through the control of the function of cDCs in the skin-draining peripheral lymph nodes (PLNs) with use of CD103-deficient mice.

\section{Results}

Deficiency of CD103 aggravates skin inflammation. To address the role of CD103 in vivo, we created mice lacking the $C d 103$ gene $\left(C d 103^{-I-}\right)$ (Supplementary Fig. 1). $C d 103^{-l-}$ mice were born at the expected Mendelian frequencies and were apparently healthy. Whereas both wild-type (WT) mice and $C d 103^{-1-}$ mice exhibited similar cellularity in skin-draining lymph nodes (PLNs), $C d 103^{-1-}$ mice exhibied the lower frequencies of cDCs and macrophages, but not other leukocytes, in spleen (Spl) than WT mice (Supplementary Figs. 2, 3). While several types of leukocytes displayed various expression level of CD103 on the cell surface in skin-draining PLNs and Spl in WT mice, their expressions were absence in $C d 103^{-1-}$ mice (Fig. 1). Upon topical application of imiquimod (IMQ), a synthetic Toll-like receptor 7 (TLR7) ligand, on the ear $\operatorname{skin}^{11,13}, C d 103^{-1-}$ mice displayed a more prominent psoriasiform dermatitis, such as thickening and scaling than WT mice (Fig. 2a,b). Histological analyses revealed that $C d 103^{-1-}$ mice showed a more significant parakeratosis, acanthosis, and Munro's microabscessess than WT mice after topical application of IMQ (Fig. 2c,d). Furthermore, $C d 103^{-1-}$ mice exhibited the prominent skin infiltration of mononuclear cells, including $\mathrm{Gr}-1^{+}$granulocytes in cutaneous inflammatory tissues when compared with WT mice upon topical application of IMQ (Fig. 2c,e,f).

Collectively, these results indicate that an absent of CD103 exacerbates psoriasiform dermatitis. 
a

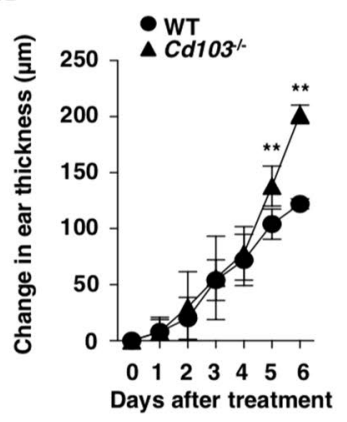

C

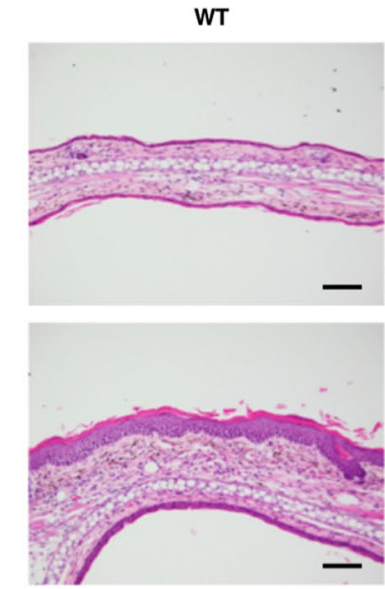

e
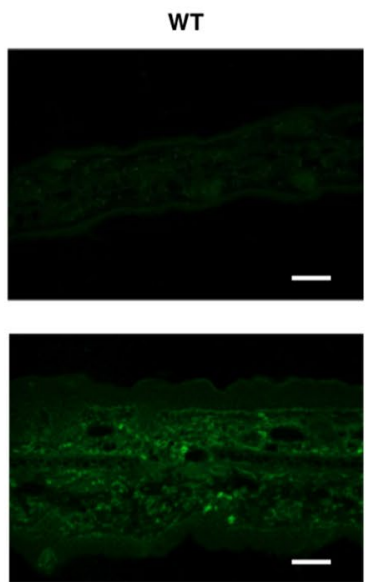

b
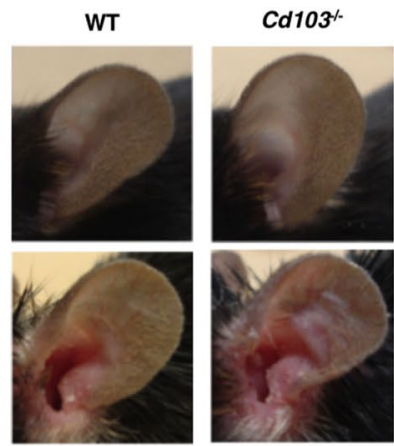

IMQ

None
Cd103-

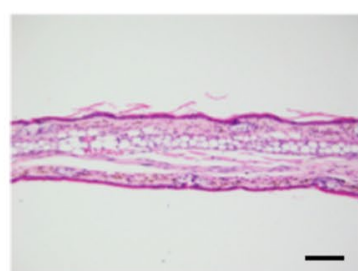

None

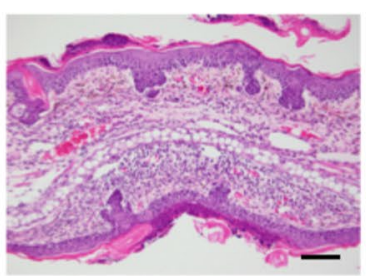

Cd103\%
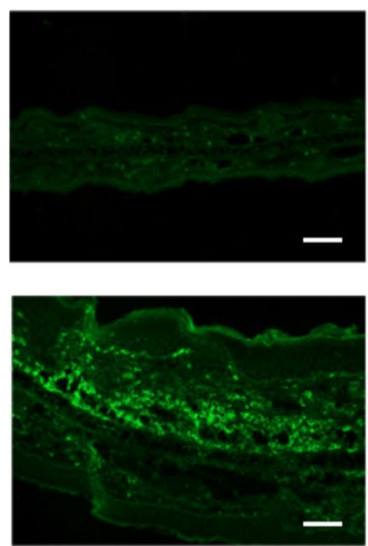

IMQ d

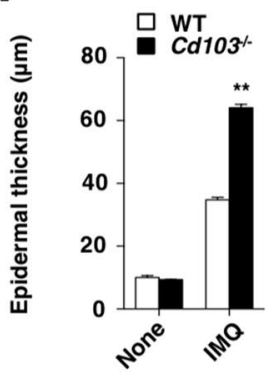

IMQ

f

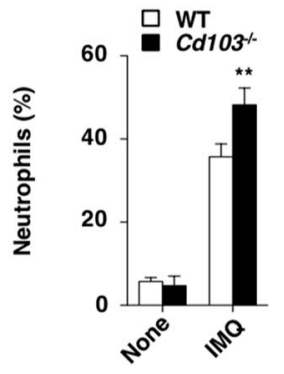

Figure 2. CD103 deficiency exacerbates psoriatic dermatitis. WT mice $(\mathrm{n}=5)$ and $C d 103^{-1-}$ mice $(\mathrm{n}=5)$ received topical treatment with IMQ on the left ear skin every day for 6 days. (a) Ear thickness was evaluated for 6 days. Data were representative of five individual samples in a single experiment. $* * P<0.01$ compared with WT mice. (b) Representative pictures of ear skin lesions at 6 days. (c,d) Hematoxylin and eosin (H\&E) sections (magnification; 20x) of ear skin at days 0 (None) and 6 (IMQ) (c), and epidermal thickness was evaluated at days 0 (None) and 6 (IMQ) (d). Data were representative of five individual samples in a single experiment. $* * P<0.01$ compared with WT mice. (e) Immunohistochemical sections (magnification; 20x) for detecting Gr-1 of ear skin at days 0 (None) and 6 (IMQ). (f) The frequency of neutrophils in ear skin at days 0 (None) and 6 (IMQ). Data are the mean \pm s.d. in four individual samples in a single experiment. $* * P<0.01$ compared with WT mice. The results are representative of at least three independent experiments with similar results.

Deficiency of CD103 accelerates skin inflammation. To clarify the role of CD103 in the regulation of psoriasiform inflammation, we examined the transcriptional expressions of cytokines and chemokines, as well as epithelial inflammation-related molecules, in psoriatic lesions in WT mice and $C d 103^{-1-}$ mice (Fig. 3). After topical application of IMQ, Cd103-/- mice exhibited higher transcriptional expressions of Illa Il1b, Il6, Il10, Il12a, Il17a, Il19, Il22, CXcl1, CXcl2, and S100a8 than WT mice. 

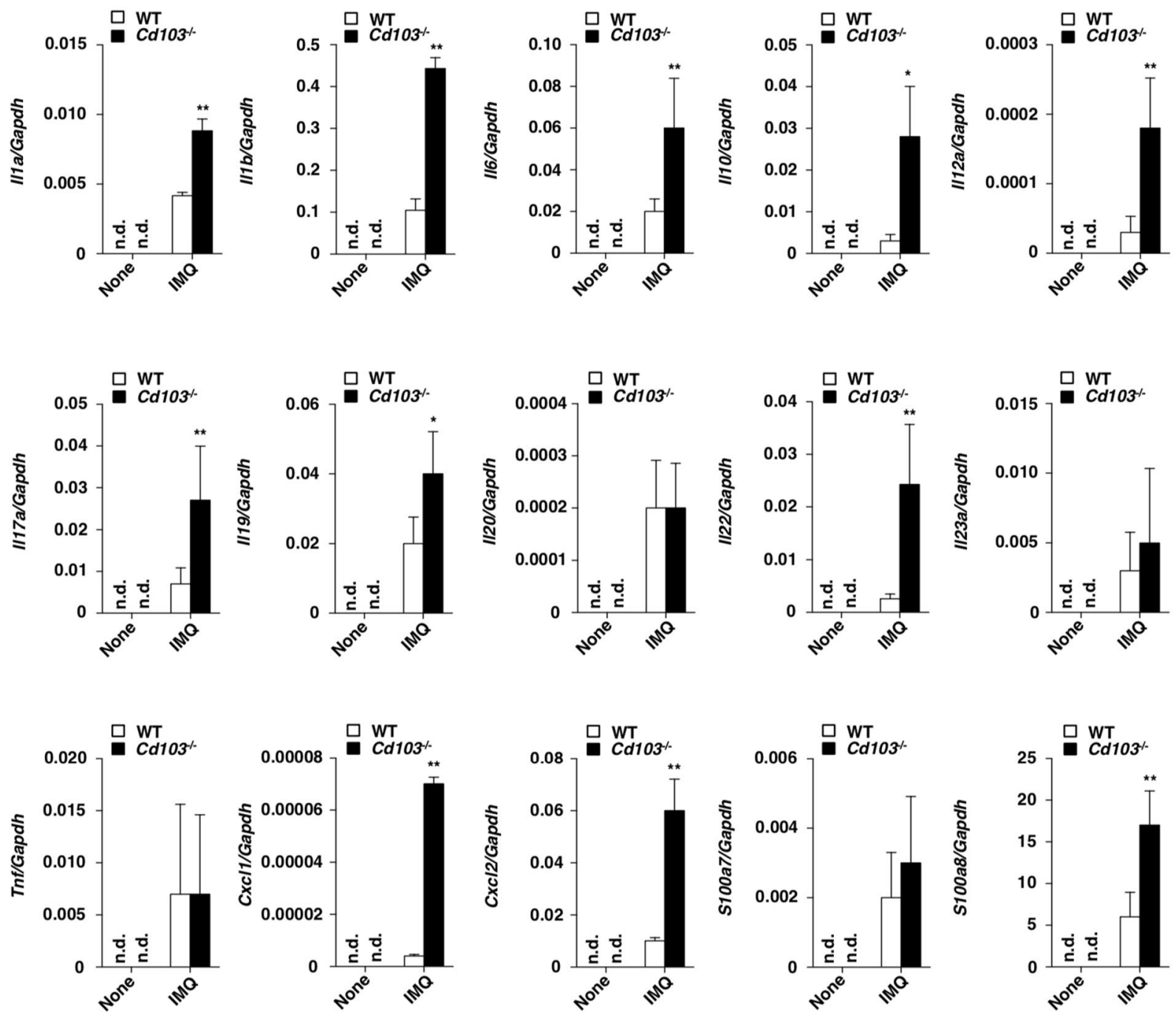

Figure 3. CD103 deficiency enhances the psoriatic inflammation. Transcriptional expressions of cytokines, chemokines, and epithelial inflammation-related molecules in ear skin at days 0 and 6 after topical application of IMQ on the ear skin in WT mice $(\mathrm{n}=3)$ and $\mathrm{Cd}_{103^{-1-}}$ mice $(\mathrm{n}=3)$, and the expression was normalized to the Gapdh transcript. Data are the mean \pm s.d. in three to five individual samples in a single experiment. $* P<0.05, * * P<0.01$ compared with WT mice. The results are representative of at least three independent experiments.

We also examined the impact of CD103 on the constituency of inflammatory leukocytes in the skin-draining PLNs after the initiation of psoriasiform dermatitis. In the skin-draining PLNs, Cd103-1- mice displayed higher or lower accumulation of migratory $\mathrm{MHC} \mathrm{II}^{\text {hi }} \mathrm{CD} 11 \mathrm{c}^{\text {med }} \mathrm{cDCs}$ (migratory $\mathrm{cDCs}$ ) and resident MHC $\mathrm{II}^{\mathrm{med}} \mathrm{CD} 11 \mathrm{c}^{\text {hi }}$ cDCs (resident cDCs), or pDCs than WT mice (Fig. 4 and Supplementary Fig. 4).

Collectively, these results indicate that a deficiency of CD103 enhances the formation of the pathogenic cytokine and leukocyte milieu to induce psoriatic inflammation.

Deficiency of CD103 enhances the activation status of CDCs. To address the influence of the absence of CD103 on the activation of cDCs, we examined the surface expressions of costimulatory molecules on cDCs in the skin-draining PLNs in psoriatic dermatitis. Upon topical treatment of IMQ on day 6, cDCs from Cd103 ${ }^{-1-}$ mice displayed marked expression levels of CD80 and CD86 as compared with those from WT mice (Fig. 5a).

We further compared the capacity of cDCs between WT mice and $C d 103^{-1-}$ mice following stimulation with IMQ. cDCs derived from skin-draining PLNs in $C d 103^{-1-}$ mice exhibited higher expressions of Il6, Il23a, and lower expressions of Il12a than those in WT mice (Fig. 5b). Similar tendency was observed in splenic cDCs in WT mice and $C d 103^{-1-}$ mice (Supplementary Fig. 5a). Furthermore, cDCs derived from skin-draining PLNs in Cd103 ${ }^{-1-}$ mice exhibited higher productions of IL- 6 and IL-12/IL-23p40 than those in WT mice (Fig. 5c). On the other hand, $c D C s$ derived from skin-draining PLNs in $C d 103^{-1-}$ mice displayed the enhanced ability to generate $\mathrm{CD}^{+} \mathrm{T}$ cells producing IL-17 ( $\mathrm{T}_{\mathrm{C}} 17$ cells) and the reduced ability of IFN- $\gamma$-producing $\mathrm{CD}^{+} \mathrm{T}$ cells $\left(\mathrm{T}_{\mathrm{C}} 1\right.$ cells) than those in WT mice (Supplementary Fig. 5b). 

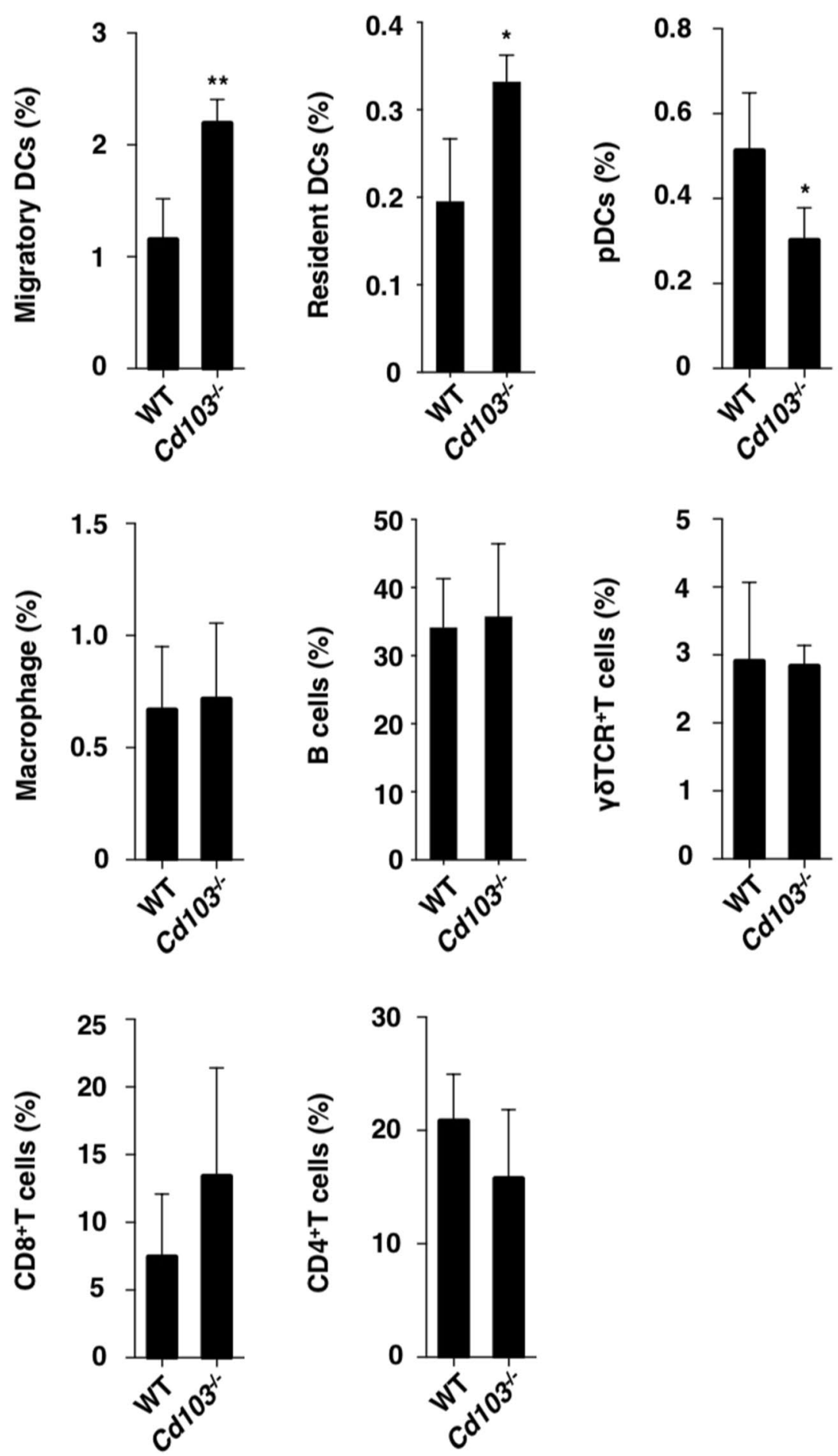

Figure 4. Absence of CD103 promotes the accumulation of inflammatory leukocytes in skin-draining PLNs. The frequency of leukocytes in the skin-draining PLNs at day 6 after topical application of IMQ on the left ear skin every day for 6 days in WT mice $(n=5)$ and $C d 103^{-1-}$ mice $(n=5)$. Data are the mean \pm s.d. in three to five individual samples in a single experiment. $* P<0.05, * * P<0.01$ compared with WT mice. The results are representative of at least three independent experiments.

Taken together, these results indicate that the absence of CD103 enhances the activation status of cDCs to generate $\mathrm{T}_{\mathrm{C}} 17$ cells.

Deficiency of CD103 promotes the differentiation of $\mathrm{T}_{\mathrm{C}} \mathbf{1 7}$ cells for the progression of psoriatic inflammation. To assess the impact of CD103 on the regulation of the axis of IL-22-IL-23-IL-17 cytokine, we compared the induction of IL-17A- and IL-22-producing lymphocytes in the skin-draining PLNs between 

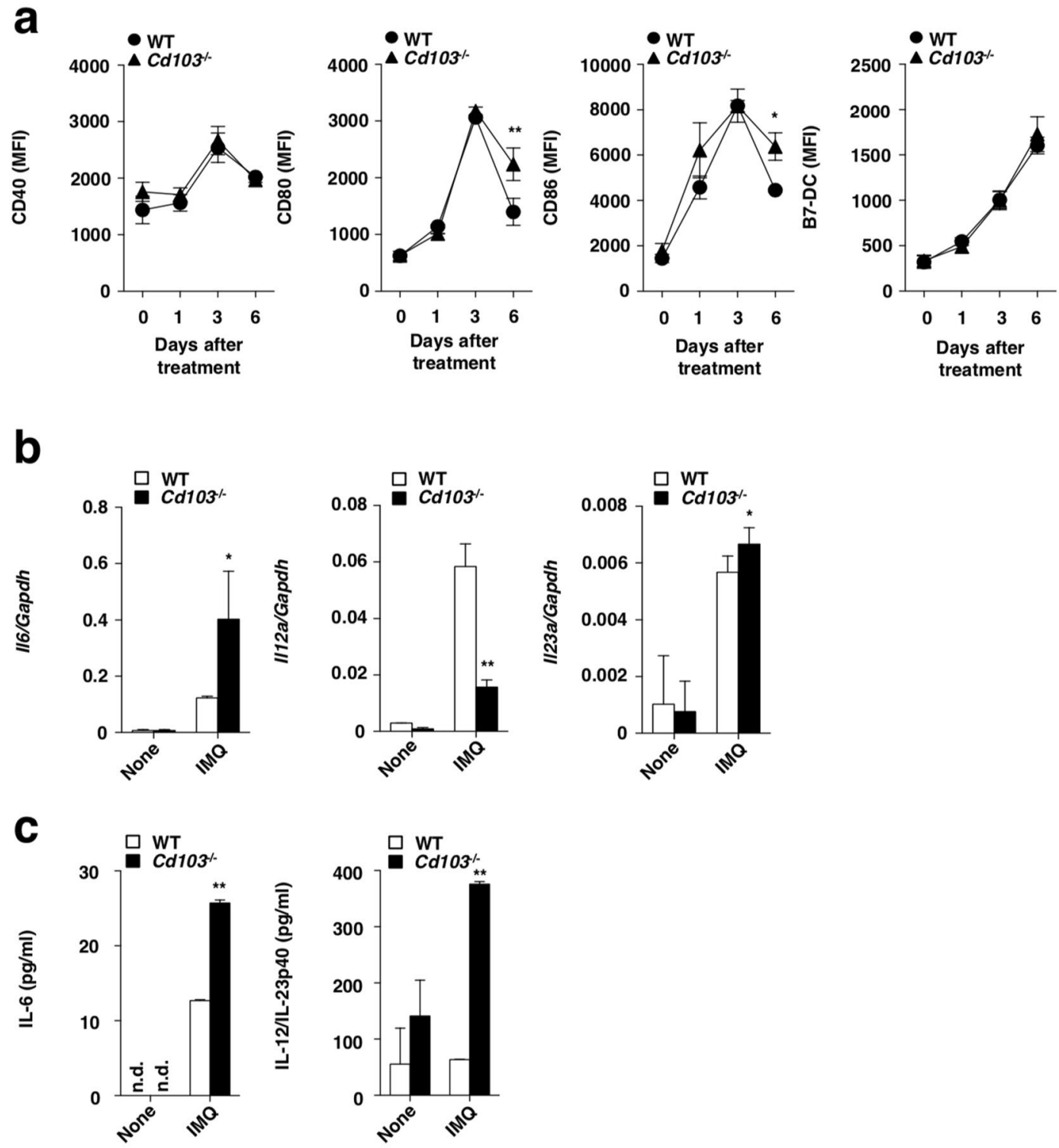

Figure 5. CD103 deficiency enhances the activation of cDCs in response to TLR7 ligand. (a) WT mice and $C d 103^{-1-}$ mice received topical treatment with or without IMQ on the left ear skin every day for 6 days. The expressions of costimulatory molecules on $\mathrm{cDCs}$ before and the indicated days after topical application of IMQ. Data are the mean fluorescence intensity $(\mathrm{MFI}) \pm$ s.d. in three individual samples in a single experiment. $* P<0.05$ compared with WT mice. $(\mathbf{b}, \mathbf{c}) \mathrm{cDCs}$ were derived from the skin-draining PLNs in WT mice and $C d 103^{-1-}$ mice were not stimulated (None) or stimulated with IMQ (IMQ). The transcriptional expressions (b) and the production $(\mathbf{c})$ of cytokines in cDCs. Data are the mean \pm s.d. in three individual samples in a single experiment. $* P<0.05, * * P<0.01$ compared with $\mathrm{WT}$ mice. The results are representative of at least three independent experiments.

WT mice and $C d 103^{-1-}$ mice following initiation of psoriasiform dermatitis. The psoriatic $C d 103^{-1-}$ mice displayed higher accumulation of IL-17A-producing ILCs and $\mathrm{T}_{\mathrm{C}} 17$ cells than the psoriatic WT mice (Fig. 6a,d, Supplementary Figs. 6a,b, and 7a,d), whereas their accumulation of other IL-17A-producing lymphocytes, such as $\gamma \delta \mathrm{TCR}^{+} \mathrm{T}$ cells and $\mathrm{T}_{\mathrm{H}} 17$ cells, were comparable (Fig. 6b,c and Supplementary Fig. 7b,c). On the other hand, there was no obvious difference in the proportions of IL-22-producing lymphocytes (Fig. 6e-h and Supplementary Fig. 7e-h). We also observed that the accumulation of IL-17A- or IL-22-producing lymphocytes in the psoriatic dermal tissues was higher in $\mathrm{Cd}_{103^{-1-}}$ mice than WT mice (Supplementary Fig. 8).

We further examined the influence of the absence of $\mathrm{CD} 103$ on the capacity of $\mathrm{CD}^{+} \mathrm{T}$ cells to differentiate into $\mathrm{T}_{\mathrm{C}} 17$ cells. $\mathrm{CD}^{+} \mathrm{T}$ cells derived from $\mathrm{Cd}_{103^{-/-}}$mice displayed an enhanced ability to develop into $\mathrm{T}_{\mathrm{C}} 17$ cells rather than $\mathrm{T}_{\mathrm{C}} 1$ cells as compared with those derived from WT mice (Supplementary Fig. 9).

Taken together, these results indicate that the deficiency of CD103 enhances the generation of IL-17A-producing lymphocytes to amplify the psoriatic inflammation. 
a

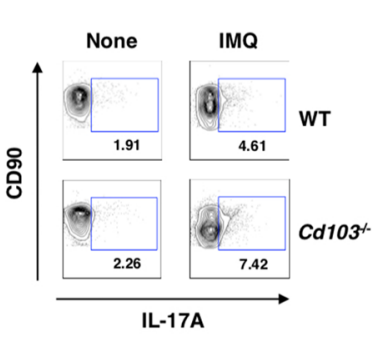

C

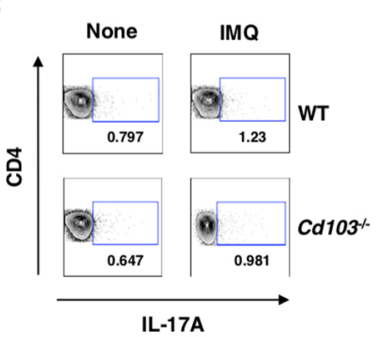

e

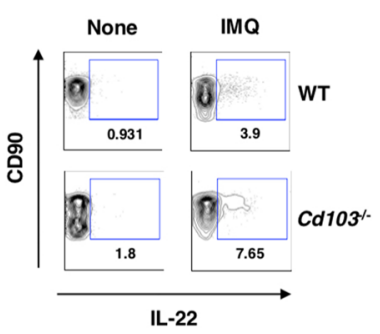

g

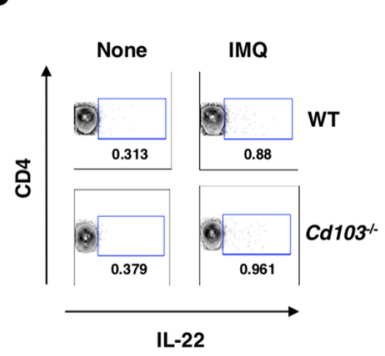

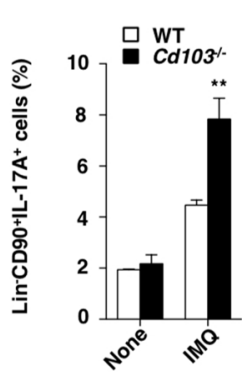
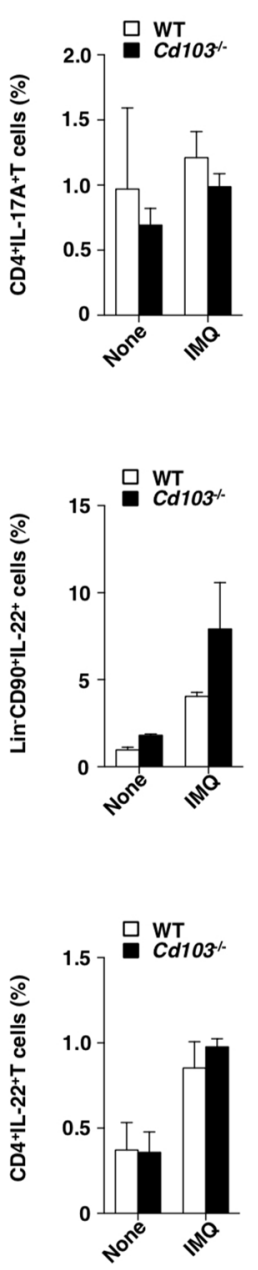

b
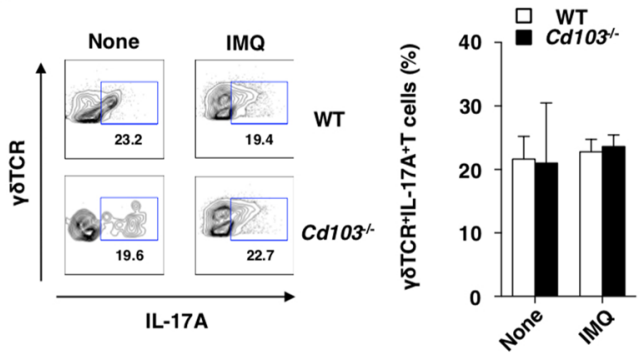

d
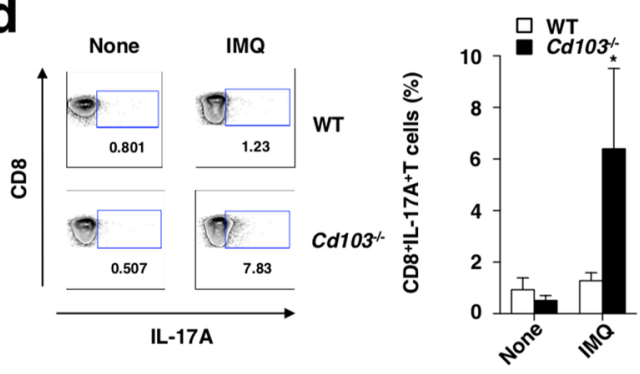

f
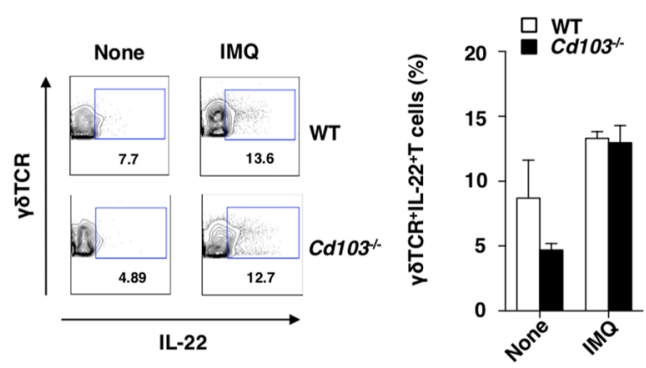

h

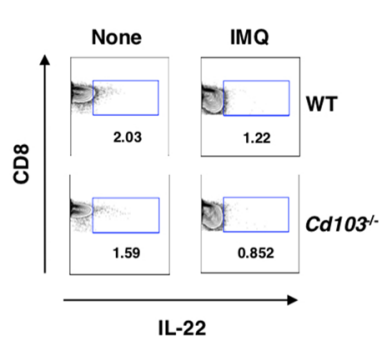

Figure 6. CD103 deficiency enhances the generation of IL-17A-prodcing lymphocytes in the skin-draining PLNs in the progression of psoriasiform skin inflammation. WT mice $(\mathrm{n}=3)$ and $C d 103^{-1-}$ mice $(\mathrm{n}=3)$ were received topical treatment with IMQ on the left ear skin every day for 6 days. The frequencies of IL17A-producing cells (a-d) and IL-22-producing cells $(\mathbf{e}-\mathbf{h})$ among innate lymphocytes $(\mathbf{a}, \mathbf{e}), \gamma \delta \mathrm{TCR}^{+} \mathrm{T}$ cells $(\mathbf{b}, \mathbf{f}), \mathrm{CD}^{+} \mathrm{T}$ cells $(\mathbf{c}, \mathbf{g})$, and $\mathrm{CD} 8^{+} \mathrm{T}$ cells $(\mathbf{d}, \mathbf{h})$ in the skin-draining PLNs at days 0 and 6 . Data are presented as a contour plot, and numbers mean the proportion of the indicated cell populations in each gate (left panel). Data are the mean \pm s.d. in three individual samples in a single experiment (right panel). $* P<0.05$, **P $P 0.01$ compared with WT mice. The results are representative of at least three independent experiments.

\section{Discussion}

Although the accumulating evidences suggest that CD103 is involved in the pathogenesis of some immune-mediated diseases such as GVHD, asthma, and allergic contact hypersensitivity, how CD103 controls immune responses leading to the development of cutaneous inflammatory disorders remains unclear. In this study, we revealed that CD103 not only inhibits the function of $\mathrm{cDCs}$ and but also impairs the intrinsic potential of $\mathrm{CD} 8^{+} \mathrm{T}$ cells to differentiate into $\mathrm{T}_{\mathrm{C}} 17$ cells to form the pathogenic cytokine and leukocyte milieu for the development of psoriasiform dermatitis.

It has been reported that CD103 is required for the localization of some lymphocytes, including CD8 $\alpha^{+}$IELs and epidermal $\gamma \delta \mathrm{TCR}^{+} \mathrm{T}$ cells, to epithelial tissues in the gut ${ }^{21}$ and skin $^{22}$. Furthermore, $\mathrm{Cd}_{103^{-1-}}$ mice on the 
$129 / \mathrm{SV} \times \mathrm{BALB} / \mathrm{c}$ background reportedly developed the cutaneous inflammatory disorder ${ }^{20}$. On the other hand, we observed that $C d 103^{-1-}$ mice on the $\mathrm{C} 57 \mathrm{BL} / 6$ background exhibited normal cellularity in skin-associated lymphoid tissues, and they did not develop the spontaneous inflammatory skin disorders under our experimental conditions. Therefore, CD103 is dispensable for the retention of lymphocytes to the skin-associated lymphoid tissues and the regulation of spontaneous inflammatory responses on the C57BL/6 background. These discrepancies might explain that CD103 controls the function of immune cells related to the immunopathogenesis depending on the context of other genetic factors and/or the environmental conditions.

While CD103 is primarily expressed by epithelial leukocytes, the potential role of CD103 in the development of psoriasiform dermatitis remains to be determined. We showed that the deficiency of CD103 aggravated the epithelial pathogenesis and the cutaneous infiltration of mononuclear cells in IMQ-induced psoriasiform dermatitis. Collectively, these results suggest that CD103 regulates the function of the inflammatory leukocytes to suppress the development of psoriasiform dermatitis. On the other hand, previous studies have shown that the deficiency of CD103 did not exacerbate skin pathogenesis in K5.hTGF 31 Tg mice. Although the reason why the roles of CD103 in the development of psoriasiform dermatitis differs in these models remain unclear, the discrepancies might be due to their distinct mechanisms underlying the initiation and progression of skin inflammation.

Although the several axes of immune dysregulation are supposed to link to the pathogenesis of psoriasiform dermatitis, how CD103 controls the immune responses leading to the cutaneous inflammation remains unclear. Our results showed that CD103 deficiency led to the enhanced productions of various inflammatory cytokines, chemokines, and antimicrobial peptides in the pathogenic skin lesions. Taken together, these results suggest that CD103 inhibits the psoriasiform skin inflammation through the suppression of the formation of the pathogenic cytokine milieu.

Although several subsets of cDCs reportedly express CD103, the role of CD103 in the control of their function remains unclear. We showed that the deficiency of CD103 enhanced the accumulation of migratory cDCs and resident $\mathrm{cDCs}$, but not other leukocytes, in the skin-draining PLNs in the development of psoriasiform dermatitis. Furthermore, the deficiency of CD103 not only enhanced the expression levels of costimulatory molecules but also augmented the secretion of IL-23. Concomitantly, the absence of CD103 enhanced the ability of cDCs to generate $T_{C} 17$ cells under $T_{C} 17$-polarized condition. Taken together, these results suggest that CD103 regulates the function of cDCs for the IL-23-mediated generation of Tc17 cells to inhibit the progression of psoriasiform dermatitis.

Whereas the IL-17A/IL-22-producing lymphocytes, known as the downstream effector cells of IL-23-IL-17 cytokine axis, are essential for the development of psoriasis ${ }^{14}$, how CD103 regulates the generation and the function of these pathogenic lymphocytes remains unclear. Our results showed that the deficiency of CD103 enhanced the generation of IL-17A-producing ILCs and $\mathrm{T}_{\mathrm{C}} 17$ cells in the skin-draining PLNs. On the other hand, the absence of $\mathrm{CD} 103$ promoted the development of $\mathrm{CD}^{+} \mathrm{T}$ cells into $\mathrm{T}_{\mathrm{C}} 17$ cells under $\mathrm{Tc} 17$-polarized culture condition, indicating that $\mathrm{CD} 103$ provide the intrinsic control of the differentiation of these pathogenic $\mathrm{CD} 8^{+} \mathrm{T}$ cells. Collectively, these results suggest that CD103 not only act on CDCs to inhibit the establishment of the milieu of IL-23-IL-17 cytokine axis but also directly impairs the ability of $\mathrm{CD}^{+}{ }^{+} \mathrm{T}$ cells to differentiate into Tc17 to ameliorate the development of psoriasiform dermatitis.

In the present study, we used $C d 103^{-/-}$mice to clarify the intrinsic role of CD103 in the development of psoriasiform dermatitis, and analysis of this strain revealed the influence of its deficiency on the functions of cDCs and $\mathrm{CD}^{+} \mathrm{T}$ cells during the disease progression. Further studies will be needed to determine the individual impact of CD103 on their function in vivo for the control of development of psoriasiform dermatitis by creating $C d 103^{\text {flox } /}$ flox mice to analyze cDC- or $\mathrm{CD} 8^{+} \mathrm{T}$ cell-specific CD103-ablation in mice.

In conclusion, we described that CD103 constitutes critical negative regulatory functions in cDCs and CD8 ${ }^{+} \mathrm{T}$ cells, it which it prevents the formation of the milieu of the pathogenic cytokine and inflammatory leukocytes for the inhibition of the development of psoriasiform dermatitis. Thus, a better understanding of precise mechanisms how CD103 regulates their functions might open new avenues for providing a new therapy for skin inflammatory immune disorders.

\section{Methods}

Mice. As described previously ${ }^{4,11,16}$, the following 6- to 12 -week-old mice were used in this study. C57BL/6 mice (Japan Clea) and B6.Cd103 $3^{-1-}$ mice as described below. All mice were bred and maintained in specific pathogen-free (SPF) conditions in the animal facility at the University of Miyazaki. All experiments were performed in accordance with institutional guidelines and approved by the Animal Experiment Committee (No. 2019-501, No. 2019-502, and No. 2019-506) and Gene Recombination Experiment Committee (No. 569).

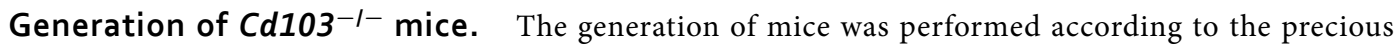
reports ${ }^{4,16}$. The targeting vector for $C d 103^{-1-}$ mice was constructed in the pBluescript vector by using a 2.0-kilobase (kb) genomic fragment (left arm) upstream of Cd103 exon 1, and a 4.0-kb genomic fragment (right arm) downstream of exon 1 cloned from a modified bacterial artificial chromosome (BAC) clone, RP23-263M10 (Children's Hospital Oakland Research Institute), containing the complete Cd103 gene. The left and right arm were custom-made using GeneArt ${ }^{\circledR}$ (Life Technologies), and each of the $5^{\prime}$ - and $3^{\prime}$-ends was tagged with XhoI and SalI sites for the left arm or SalI and ClaI sites for the right arm, respectively. Following the digestion of the $2.0-\mathrm{kb}$ fragment with $X$ hoI and SalI, and the 4.0-kb fragment with SalI and ClaI, each fragment was ligated into each site of pBluescript. A SalI restriction site was engineered in place of the start codon in exon 1 . The FRT-PGK-gb2-Neo-FRT-Stop cassette was cloned into the SalI site inserted into the targeting vector. Finally, the targeting construct was abutted to a MC1-DTa negative-selection cassette and linearized. The linearized targeting construct was introduced by electroporation into C57BL/6-derived JN/2 recombinant embryonic stem cell (ESC) and neomycin-resistant clones were first screened for homologous recombination by PCR utilizing a pair 
of the following oligonucleotides: Primer 1 (5'-ATA TGT AGT GTC TGG TCA GGA TAA TAG TTG-3') and Primer 2 (5'-ATA ACC TCC TCT CCT ATG GTA CCT AAA C-3'). SpeI-digested genomic DNA of positive clones was then screened by Southern blotting with a $3^{\prime}$ external single-copy probe corresponding to a $0.607-\mathrm{kb}$ fragment, which was amplified by PCR using these primers: 5'-TAT ACA CAC CTA TGA ATG CAT GCT C- $3^{\prime}$ and 5'-CTC TGA CTA AAC CCC ATC TTG ATA A-3'. When tested on SpeI-digested DNA, it hybridized either to a 13.3-kb WT fragment or to a $10.7-\mathrm{kb}$ recombinant fragment. ESC clones bearing the correctly targeted locus were injected into $\mathrm{BALB} / \mathrm{c}$ blastocysts, and chimeric male offspring were mated with female C57BL/6 mice to obtain heterozygotes, which were then crossed to obtain homozygotes. Transmission of the targeted allele was confirmed by PCR with Primer 1 and Primer 3 (5'-CTT TAT ATT TCA TTT TTG CTC AGG CTT C-3'). The mutant mice were cross-mated for more than nine generations with B6.FLIP mice to excise the flanked FRT sites by Flp-recombinase, and 8- to 12 -week-old $C d 103^{+/+}$littermates were used as WT mice. Then, $C d 103^{+/-}$littermates were crossed to obtain homozygotes, and transmission of the targeted allele was confirmed by PCR with Primer 1 and Primer 3.

Tissues and cell isolation. Isolations of tissue and cells were performed according to the precious reports $^{4,11,16}$. To prepare single-cell suspensions from Spl or PLNs were digested with $400 \mathrm{U} / \mathrm{ml}$ collagenase type III (Worthington Biochemical) at $37^{\circ} \mathrm{C}$ for $20 \mathrm{~min}$ or $30 \mathrm{~min}$ and were ground between glass slides. Cell suspensions of Spl were further treated with RBC lysis buffer (Sigma-Aldrich). Ear skin fragments were dissected and digested with collagenase type III at $60 \mathrm{~min}$ as described above. Single-cell suspensions were obtained by forcing through a $100-\mu \mathrm{m}$ cell strainer (BD Biosciences). CD11 $\mathrm{c}^{+}$DCs were purified by AutoMACS with mouse CD11c (N418) Microbeads. CD3 ${ }^{+} \mathrm{CD}^{+} \mathrm{T}$ cells were purified from splenocytes with mouse CD8 T-lymphocyte Enrichment Set-DM (BD Biosciences).

Flow cytometry. Flow cytometry were performed according to the precious reports ${ }^{4,11,16}$. Cells were stained with fluorescein-conjugated mAbs and Ab to mouse, CD4 (RM4-5), CD8 $\alpha$ (53-6.7), CD11c (HL3), CD40 (3/23), CD45R/B220 (RA3-6B2), CD45.2 (104), CD80 (16-10A1), CD86 (GL1), CD103 (M290), I-A/I-E (M5/114.15.2), Gr-1 (RB6-8C5), IL-17A (TC11-18H10), isotype-matched control mAb (cont. Ig) (BD Biosciences), CD3E (145-

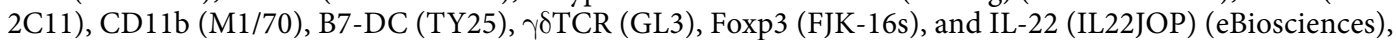
CD90.2 (53-2.1), F4/80 (BM8), Siglec-H (551) (BioLegend). For the intracellular expression of cytokines, cells were incubated for $4 \mathrm{hrs}$ with phorbol 12-myristate 13-acetate (PMA, $50 \mathrm{ng} / \mathrm{ml}$; Sigma-Aldrich) and ionomycin ( $500 \mathrm{ng} / \mathrm{ml}$; Sigma-Aldrich) plus Brefeldin A (eBiosciences) during the final $2 \mathrm{hrs}$. Subsequently, the cells were resuspended in Fixation-Permeabilization solution (eBiosciences) and intracellular cytokine staining was carried out according to the manufacturer's directions. Fluorescence staining was analyzed with a FACSVerse flow cytometer and FlowJo software (both from BD Biosciences).

Quantitative reverse transcriptase polymerase chain reaction (qRT-PCR). qPCR was performed according to the precious reports ${ }^{11,16}$. Total RNA from ear skin was extracted with ISOGEN II (NIPPON GENE) and the first-strand complementary DNA (cDNA) was synthesized from $1 \mu \mathrm{g}$ of total RNA using the PrimeScript RT Master Mix (Takara) according to the manufacturer's instructions. Transcriptional expression levels were analyzed by using SYBR ${ }^{\circledR}$ Premix Ex Taq II on Thermal Cycler Dice (Takara) with specific primer pairs (Supplementary Table 1) after normalization for the expression of Gapdh.

Skin inflammation. Induction and analysis of IMQ-induced psoriasiform skin inflammation were performed according to the precious reports ${ }^{11,16}$. Mice were treated topically with either $25 \mathrm{mg}$ of $5 \%$ IMQ cream (Mochida Pharmaceutical) or petrolatum (Wako Pure Chemicals) as a control on the left ear every day for 6 days. The severity of the ear skin inflammation of each mouse was determined daily by ear thickness using digital calipers (PK-1012CPX; Mitsutoyo), and ear photographs were taken at days 0 and 6 after topical application of IMQ cream on the ear skin.

Histopathologic assessment. Assessment of the Histopathology was performed according to the precious reports $^{11,16}$. Ear tissues were fixed with $4 \%$ paraformaldehyde (PFA) in PBS and embedded in paraffin. The tissue sections ( $5 \mu \mathrm{m}$ thickness) were stained with H\&E. The stained slides were examined with a bright-field microscope (BX53; Olympus). The thickness of ear epidermis and dermis was measured for thickness using ImageJ (National Institutes of Health) by a blinded observer.

Immunohistochemical analysis. Immunohistochemical analysis was performed according to the precious reports ${ }^{11,16}$. For cutaneous detection of Gr-1 $1^{+}$granulocytes $^{11,16}$, the ear skin was embedded in OCT compound (Sakura Fineteck) and frozen in liquid $\mathrm{N}_{2}$. The tissue block was sectioned with a cryostat at 5-7 $\mu \mathrm{m}$. Frozen sections were fixed with cold acetone and blocked in PBS containing 5\% normal rat serum. Subsequently, slide was stained with FITC-conjugated anti-Gr-1 mAb (BD Biosciences) and mounted with Vectashield (Vector laboratories). The stained slides were analyzed with a All-in-One Fluorescence Microscope (BZX-710; KEYENCE).

Culture of DCs. According to the previous reports ${ }^{11,16}, \mathrm{DCs}\left(10^{5}\right)$ were cultured with or without IMQ $(10 \mu \mathrm{g} /$ $\mathrm{ml}$; Invivogen) for $5 \mathrm{hrs}$ in 96-well flat-bottomed plates (BD Bioscience).

In vitro $\mathrm{T}_{\mathrm{C}} \mathbf{1 7}$ cell differentiation assay. As described in previous reports with some modification ${ }^{4,11,16}$, naïve CD8 ${ }^{+} \mathrm{T}$ cells $\left(5 \times 10^{4}\right)$ were cultured with cDCs $\left(2.5 \times 10^{4}\right)$ in combination with anti-CD3 $\mathrm{mAb}(1$ $\mu \mathrm{g} / \mathrm{ml} ; 145-2 \mathrm{C} 11$, Biolegend), anti-IFN- $\gamma \mathrm{mAb}(5 \mu \mathrm{g} / \mathrm{ml}$; R4-6A2, BD Biosciences), recombinant human transforming growth factor (TGF)- $\beta$ ( $2 \mathrm{ng} / \mathrm{ml}$; Wako Pure Chemicals) and IL-6 (40 ng/ml) for 5 days in 96 -well round-bottomed plates (BD Bioscience). In another experiment, naïve $\mathrm{CD} 8^{+} \mathrm{T}$ cells $\left(4 \times 10^{4}\right)$ were cultured in 
combination with anti-CD3 $\varepsilon \mathrm{mAb}(1 \mu \mathrm{g} / \mathrm{ml})$, anti-CD28 mAb $(1 \mu \mathrm{g} / \mathrm{ml})$, anti-IFN- $\gamma \mathrm{mAb}(5 \mu \mathrm{g} / \mathrm{ml})$, recombinant human TGF- $\beta 1(2 \mathrm{ng} / \mathrm{ml})$ and IL-6 $(20 \mathrm{ng} / \mathrm{ml})$ for 5 days in 96-well flat-bottomed plate. Analysis of IL-17A and IFN- $\gamma$ expression among gated $\mathrm{CD} 8^{+} \mathrm{T}$ cells was performed by flow cytometry as described above.

Statistical analysis. Statistical analysis was performed as described previously ${ }^{4,11,16}$. Data are the means \pm s.d. from three to eight individual samples in a single experiment, and we performed at least four independent experiments. The statistical significance of differences between groups was analyzed using Student's $t$-test (two-tailed). P values $<0.05$ or 0.01 were considered statistically significant.

Received: 25 September 2019; Accepted: 30 April 2020;

Published online: 20 May 2020

\section{References}

1. Guilliams, M. et al. Dendritic cells, monocytes and macrophages: a unified nomenclature based on ontogeny. Nat. Rev. Immunol. 14, 571-578 (2014)

2. Murphy, T. L. et al. Transcriptional control of dendritic cell development. Annu. Rev. Immunol. 34, 93-119 (2016).

3. Sato, K., Uto, T., Fukaya, T. \& Takagi, H. Regulatory dendritic cells. Curr. Top. Microbiol. Immunol. 410, 47-71 (2017).

4. Takagi, H. et al. Plasmacytoid dendritic cells are crucial for the initiation of inflammation and T cell immunity in vivo. Immunity 35, 958-971 (2011).

5. Pasparakis, M., Haase, I. \& Nestle, F. O. Mechanisms regulating skin immunity and inflammation. Nat. Rev. Immunol. 14, 289-301 (2014).

6. Lowes, M. A., Suárez-Fariñas, M. \& Krueger, J. G. Immunology of psoriasis. Annu. Rev. Immunol. 32, 227-255 (2014).

7. Gilliet, M. \& Lande, R. Antimicrobial peptides and self-DNA in autoimmune skin inflammation. Curr. Opin. Immunol. 20, 401-407 (2008).

8. Lai, Y. et al. The antimicrobial protein REG3A regulates keratinocyte proliferation and differentiation after skin injury. Immunity 37, 74-84 (2012).

9. Cai, Y. et al. Pivotal role of dermal IL-17-producing $\gamma \delta ~ T$ cells in skin inflammation. Immunity 35, 596-610 (2011).

10. Glitzner, E. et al. Specific roles for dendritic cell subsets during initiation and progression of psoriasis. EMBO Mol. Med. 6 , 1312-1327 (2014).

11. Takagi, H. et al. Plasmacytoid dendritic cells orchestrate TLR7-mediated innate and adaptive immunity for the initiation of autoimmune inflammation. Sci. Rep. 6, 24477 (2016).

12. Kumari, S. et al. Tumor necrosis factor receptor signaling in keratinocytes triggers interleukin-24-dependent psoriasis-like skin inflammation in mice. Immunity 39, 899-911 (2013).

13. van der Fits, L. et al. Imiquimod-induced psoriasis-like skin inflammation in mice is mediated via the IL-23/IL-17 axis. J. Immunol. 182, 5836-5845 (2009).

14. Lowes, M. A. et al. The IL-23/T17 pathogenic axis in psoriasis is amplified by keratinocyte responses. Trends Immunol. 34, 174-181 (2013).

15. Pantelyushin, S. et al. Ror $\gamma \mathrm{t}^{+}$innate lymphocytes and $\gamma \delta \mathrm{T}$ cells initiate psoriasiform plaque formation in mice. J. Clin. Invest. 122, 2252-2256 (2012).

16. Fukaya, T. et al. Pivotal role of IL-22 binding protein in the epithelial autoregulation of interleukin-22 signaling in the control of skin inflammation. Front. Immunol. 9, 1418 (2018).

17. Cepek, K. L. et al. Adhesion between epithelial cells and Tlymphocytes mediated by E-cadherin and the $\alpha \mathrm{E} \beta 7$ integrin. Nature 372, 190-103 (1994).

18. Karecla, P. I., Bowden, S. J., Green, S. J. \& Kilshaw, P. J. Recognition of E-cadherin on epithelial cells by the mucosal T cell integrin

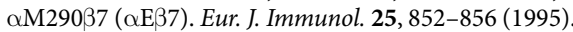

19. Kilshaw, P. J. \& Higgins, J. M. Alpha E: no more rejection? J. Exp. Med. 19, 873-875 (2002).

20. Schön, M. P. et al. Cutaneous inflammatory disorder in integrin $\alpha \mathrm{E}$ (CD103)-deficient mice. J. Immunol. 165, 6583-6589 (2000).

21. Schön, M. P. et al. Mucosal T lymphocyte numbers are selectively reduced in integrin $\alpha \mathrm{E}$ (CD103)-deficient mice. J Immunol. 162, 6641-6649 (1999).

22. Schön, M. P., Schön, M., Parker, C. M. \& Williams, I. R. Dendritic epidermal T cells (DETC) are diminished in integrin $\alpha E(C D 103)-$ deficient mice. J. Invest. Dermatol. 119, 190-193 (2002).

23. Feng, Y. et al. CD103 expression is required for destruction of pancreatic islet allografts by CD8 ${ }^{+}$T cells. J. Exp. Med. 196, 877-886 (2002).

24. El-Asady, R. et al. TGF- $\beta$-dependent CD103 expression by $\mathrm{CD} 8^{+} \mathrm{T}$ cells promotes selective destruction of the host intestinal epithelium during graft-versus-host disease. J. Exp. Med. 201, 1647-1657 (2005).

25. Bernatchez, E. et al. Pulmonary CD103 expression regulates airway inflammation in asthma. Am. J. Physiol. Lung Cell. Mol. Physiol. 308, L816-L826 (2015).

26. Fousteri, G. et al. Minimal effect of CD103 expression on the control of a chronic antiviral immune response. Viral Immunol. 23, 285-294 (2010).

27. Mullaly, S. C., Burrows, K., Antignano, F. \& Zaph, C. Assessing the role of CD103 in immunity to an intestinal helminth parasite. PLoS One 6, e19580 (2011).

28. Braun, A. et al. Integrin $\alpha \mathrm{E}(\mathrm{CD} 103)$ is involved in regulatory T-cell function in allergic contact hypersensitivity. J. Invest. Dermatol. 135, 2982-2991 (2015).

29. Braun, A. et al. Integrin $\alpha \mathrm{E}(\mathrm{CD} 103)$ is induced but plays no pathogenic role in psoriasiform skin lesions of TGF $\beta 1$ transgenic mice. Exp. Dermatol. 25, 311-313 (2016).

\section{Acknowledgements}

We thank all members of the animal facility at University of Miyazaki; Yumiko Sato and Ikumi Goto for secretarial assistance and Yukari Kawagoe for technical help in cell sorting. This work was supported by a Grant-in-Aid for Scientific Research (B) (K.S.; 18H02670), for challenging Exploratory Research (K.S.; 16K15291), and for Young Scientists (B) (T.U.; 17K15027, T.Fukaya.; 17K15732, and H.T.; 18K15194) from the Ministry of Education, Science and Culture of Japan, the Project for Cancer Research And Therapeutic Evolution (P-CREATE) from Japan Agency for Medical Research and development (AMED) (K.S.; 16cm0106307h0001, 17cm0106307h0002, $18 \mathrm{~cm} 0106307 \mathrm{~h} 0003$, and $19 \mathrm{~cm} 0106307 \mathrm{~h} 0004)$, the Uehara Memorial Foundation (H.T.), Takeda Science Foundation (T.U. and T.Fukaya.), the Naito Foundation (K.S.), Bristol-Myers Squibb Foundation Grants (K.S.), GSK Japan Research Grant 2016 (T.Fukaya.), GSK Japan Research Grant 2017 (H.T.), GSK Japan Research Grant 
2018 (T.U.), Daiichi Sankyo Foundation of Life Science (K.S.), Nipponham Foundation for the Future of Food (H.T.), and the Shin-Nihon Foundation of Advanced Medical Research (T.U.).

\section{Author contributions}

K.S. designed all experiments, analyzed data and wrote the manuscript, T.Fukui, T.Fukaya, T.U., H.T., J.N., N.M., Y.N. and N.C. performed experiments, and H. K., Y.H. and Y.Y. provided reagents and information.

\section{Competing interests}

The authors declare no competing interests.

\section{Additional information}

Supplementary information is available for this paper at https://doi.org/10.1038/s41598-020-65355-9.

Correspondence and requests for materials should be addressed to K.S.

Reprints and permissions information is available at www.nature.com/reprints.

Publisher's note Springer Nature remains neutral with regard to jurisdictional claims in published maps and institutional affiliations.

(c) (i) Open Access This article is licensed under a Creative Commons Attribution 4.0 International License, which permits use, sharing, adaptation, distribution and reproduction in any medium or format, as long as you give appropriate credit to the original author(s) and the source, provide a link to the Creative Commons license, and indicate if changes were made. The images or other third party material in this article are included in the article's Creative Commons license, unless indicated otherwise in a credit line to the material. If material is not included in the article's Creative Commons license and your intended use is not permitted by statutory regulation or exceeds the permitted use, you will need to obtain permission directly from the copyright holder. To view a copy of this license, visit http://creativecommons.org/licenses/by/4.0/.

(c) The Author(s) 2020 